their HIV status decreased from $51 \%$ in 2006 to 32\% in 2011 ( $p<0.05)$. Between 2006 and 2011, STI positivity rate increased from $17.4 \%$ to $20.6 \%$ ( $p<0.05$ ), caused by a significant increase in positivity rate for chlamydia $(9.5 \%$ to $11.0 \%)$ and gonorrhoea $(8.4 \%$ to $10.1 \%)$. Syphilis positivity rate decreased significantly over time, HIV remained stable.

In multivariable analyses, factors significantly associated with an STI were being notified (OR:2.8; 95\% CI: 2.5-3.2), multiple sex partners (OR:2.4; 95\% CI: 2.0-3.0), previous STI (OR:1.9; 95\% CI: 1.6-2.1) and being involved in sex work (OR:1.5; 95\% CI: 1.2-1.9). In addition, non-Dutch young MSM were at significantly higher risk for an STI, as were homosexual men compared to bisexual men (OR: 1.3; 95\% CI: 1.1-1.4).

Conclusions Since the number of consultations and the proportion aware of their HIV status increased over time, awareness for STI seems to be increasing in young MSM. However, gonorrhoea and chlamydia positivity rates are still increasing. Therefore special attention needs to be maintained towards counselling and reaching specific high-risk sub-groups, including young migrant MSM and young MSM involved in sex work to limit on-going transmission of STI.

\section{P3.158 NEWLY IDENTIFIED HIV INFECTION AMONG PATIENTS DIAGNOSED WITH EARLY SYPHILIS, CHICAGO, IL, 2006-2011}

doi:10.1136/sextrans-2013-051184.0616

I Tabidze, R Hazen, N Benbow. Department of Public Health, Chicago, IL, United States

Background Concomitant HIV and syphilis infections are prevalent among the same risk groups. Increases in syphilis cases among men who have sex with men (MSM) have been characterised by high rates of HIV co-infection. Our objectives were to compare demographic characteristics and percentage of persons diagnosed with Early syphilis (ES) and co-infected with HIV between 2006-2011.

Methods Surveillance data for ES (Primary, Secondary, Early Latent stages) and HIV cases reported to the Chicago Department of Public Health (CPDH) were analysed retrospectively using SAS version 9.3(2). Newly identified HIV infection was defined by earliest diagnosis date of HIV in the Enhanced HIV/AIDS Reporting System (eHars).

Results Between 2006-2011, there were 4,542 reported ES cases among 3,929 individuals; $40 \%(1,562 / 3,929)$ of individuals were matched to records in eHars. A total of 735 HIV infections occurred from 2006-2011: 52\% (384) were co-infected from 2006-2008 and $48 \%$ (351) from 2009-2011. Despite decline in the number of HIV infections after syphilis diagnosis from 384 to 351 (-8.6\%), the proportion of co-infected MSM from 2006-2008 and 2009-2011 remained stable at $92 \%$ and $91 \%$, respectively. By race, the proportion of co-infected Black MSM increased from $54.2 \%$ to $57.1 \%$, while the proportion of Whites remained stable $(24.2 \%$ and $25.2 \%$, respectively) and Hispanics declined $(15.4 \%$ and $12.1 \%$, respectively). Despite declines by race for most age categories, the number of co-infected Black MSM ages 13-24 increased by $29.2 \%$ and the number of co-infected White MSM ages 45-54 increased by $75 \%$.

Conclusions Despite an overall decline in HIV co-infections, the proportion of co-infection remained stable among MSM with an increase in the percentages in HIV sero-prevalence among Black MSM ages 13-24 and White MSM age 45-54, identifying them as a critical target group for STI/HIV prevention efforts.

\section{P3.159 IDENTIFYING SYPHILIS RISK NETWORKS THROUGH VENUE ATTENDANCE IN SAN FRANCISCO}

doi:10.1136/sextrans-2013-051184.0617

'S Stephens, 'C Fann, ${ }^{2} \mathrm{~F}$ Strona, ${ }^{2} \mathrm{~W}$ Wolf, ${ }^{1} \mathrm{~S}$ Cohen, ${ }^{1 S}$ Philip, ${ }^{1} \mathrm{~K}$ Bernstein. ${ }^{1}$ San Francisco Department of Public Health, San Francisco, CA, United States; ${ }^{2}$ Centers for Disease Control and Prevention, Atlanta, GA, United States
Background Many men who have sex with men (MSM) interviewed through syphilis partner services report large numbers of sex partners but can provide contact information for relatively few. Prioritizing interventions for patients with syphilis who are part of large sexual networks may be "high yield" and identifying venues named by syphilis cases who report high numbers of partners may help identify such networks. We compared syphilis patients across three levels of sexual partner frequency.

Methods For each venue reported by interviewed patients with early syphilis in 2011, we examined the distribution of total reported sex partners (not only named partners) in the last year. Based on the median number of total partners among men who named each venue, we categorised venues into three levels of partner frequency: high (> 15 partners reported), medium (6-15 partners reported), and low ( $<6$ partners reported). Interviewed early syphilis cases were then classified as attending high, medium, or low partner frequency venues; sociodemographic and risk behaviours were compared across the three venue categories using $\mathrm{X}^{2}$ tests.

Results In 2011, 433 patients with early syphilis named 32 venues. One hundred forty three (32.3\%) patients were categorised as high partner frequency venue users, $226(51.0 \%)$ as medium partner frequency venue users, and $74(16.7 \%)$ as low partner frequency-only venue users. Patients with early syphilis that reported meeting partners at high-frequency venues were generally older, more likely to be white, have a previous syphilis infection, use methamphetamines in the previous year, and be HIV-infected (all $p<0.05$ ) than those who reported meeting partners at medium-frequency and low-frequency venues.

Discussion Venues where partners are met may be an appropriate proxy for network membership. Targeting additional resources, outreach, and services to clients who attend high frequency venues may have a positive impact on syphilis prevention efforts.

\section{P3.160 HIGH RISK SEXUAL BEHAVIOURS AND SEXUALLY TRANSMITTED INFECTIONS AMONG TEENAGE MEN WHO HAVE SEX WITH MEN}

doi:10.1136/sextrans-2013-051184.0618

${ }^{1}$ H Zou, ${ }^{* 1,2} \mathrm{C}$ Fairley, ${ }^{3} \mathrm{~A}$ Grulich, ${ }^{4} \mathrm{~S}$ Garland, ${ }^{2} \mathrm{~J}$ Sze, ${ }^{2} \mathrm{~A}$ Afrizal, ${ }^{* 1,2} \mathrm{M}$ Chen. ${ }^{1}$ School of Population Health, University of Melbourne, Carlton, Australia; ${ }^{2}$ Melbourne Sexual Health Centre, Alfred Health, Carlton, Australia; ${ }^{3}$ Kirby Institute, University of New South UK, Sydney, Australia; ${ }^{4}$ Department of Obstetrics and Gynaecology, University of Melbourne, Parkville, Australia

*Joint last authors

Background Data on testing and detection of sexually transmissible infections (STIs) in younger MSM are scarce and no specific study focusing on teenage MSM has been published. In this study we report on sexual risk behaviours and STI testing and detection in teenage MSM aged 15-20 attending a sexual health service.

Methods Data were extracted from Melbourne Sexual Health Centre's computerised medical records system on self-reported sexual behaviours and test results between July 2008 and June 2012 Results for MSM aged 15-20 were compared with those of older MSM.

Results 445 MSM aged 15-20 and 4313 MSM aged 20 or older were included. The median number of casual male partners in the past 12 months was 4 and 5 ( $p=0.015)$ for teenage and older MSM, respectively. Compared to older MSM, Teenage MSM were less likely to participate in insertive anal sex $(91.9 \%$ vs $86.8 \%, p=0.002)$ and more likely to participate in receptive anal sex ( $86.3 \%$ vs $92.4 \%$, $\mathrm{p}=0.002)$ with casual male partners. Teenage MSM were more likely to consistently use a condom in insertive anal sex $(39.0 \%$ vs $32.7 \%, p=0.024$ ) with regular partners but less likely to consistently use a condom inwith receptive anal sex with casual male partners $(45.5 \%$ vs $56.6 \%, \mathrm{p}=0.001)$. 